\title{
Complementation between inactive fragments of Sssl DNA methyltransferase
}

Krystyna Ślaska-Kiss, Edit Tímár and Antal Kiss*

\begin{abstract}
Background: Silencing mammalian genes by targeted DNA (cytosine-5) methylation of selected CG sites in the genome would be a powerful technique to analyze epigenomic information and to study the roles of DNA methylation in physiological and pathological states. A promising approach of targeted DNA methylation is based on the ability of split fragments of a monomeric DNA methyltransferase (C5-MTase) to associate and form active enzyme. A few C5-MTases of different specificities have been shown to possess the ability of fragment complementation, but a demonstration of this phenomenon for a C5-MTase, which has CG specificity and thus can be targeted to methylate any CG site, has been lacking. The purpose of this study was to test whether the CG-specific prokaryotic C5-MTase M.Sssl shows the phenomenon of fragment complementation.

Results: We show that truncated inactive N-terminal fragments of M.Sssl can assemble with truncated inactive C-terminal fragments to form active enzyme in vivo when produced in the same E. coli cell. Overlapping and non-overlapping fragments as well as fragments containing short appended foreign sequences had complementation capacity. In optimal combinations C-terminal fragments started between conserved motif VIII and the predicted target recognizing domain of M.Sssl. DNA methyltransferase activity in crude extracts of cells with the best complementing fragment pairs was $\sim 4$ per cent of the activity of cells producing the full length enzyme. Fusions of two N-terminal and two C-terminal fragments to $21.6 \mathrm{kDa}$ zinc finger domains only slightly reduced complementation ability of the fragments.

Conclusions: The CG-specific DNA methyltransferase M.Sssl shows the phenomenon of fragment complementation in vivo in E. coli. Fusion of the split fragments to six unit zinc finger domains does not substantially interfere with the formation of active enzyme. These observations and the large number of complementing fragment combinations representing a wide range of MTase activity offer the possibility to develop M.Sssl into a programmable DNA methyltransferase of high specificity.
\end{abstract}

Keywords: Sssl DNA methyltransferase, DNA methylation, 5-methylcytosine, Protein fragment complementation, Protein fusion, Zinc finger

\section{Background}

Cytosine C5-methylation plays important roles in several biological phenomena, such as restriction-modification in prokaryotes, genomic imprinting and carcinogenesis in eukaryotes [1]. This reaction is catalyzed by DNA (cytosine-5) methyltransferases (C5-MTase), which transfer a methyl group from $\mathrm{S}$-adenosyl-methionine (AdoMet) to carbon 5 of cytosine in specific DNA sequences [2]. Prokaryotic C5-MTases contain ten

\footnotetext{
* Correspondence: kiss.antal@brc.mta.hu

Institute of Biochemistry, Biological Research Center of the Hungarian

Academy of Sciences, Temesvári krt. 62, 6726 Szeged, Hungary
}

conserved sequence motifs and a so-called variable region located between conserved motifs VIII and IX [3]. The conserved motifs are responsible for the general steps of the methyl transfer reaction [4-9], whereas specific sequence recognition is mediated mainly by the variable region [10] [11]. Our understanding of the three-dimensional structure of C5-MTases and of their interaction with substrate DNA is mainly based on the X-ray structures of two enzymes: M.HhaI and M.HaeIII. They revealed that both MTases fold in two domains, the large domain encompassing most of the conserved motifs and the small domain containing the variable region and conserved motif IX. The two domains form a

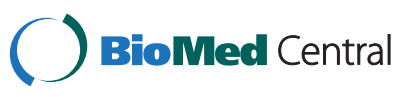

(c) 2012 Slaska-Kiss et al.; licensee BioMed Central Ltd. This is an Open Access article distributed under the terms of the Creative Commons Attribution License (http://creativecommons.org/licenses/by/2.0), which permits unrestricted use, distribution, and reproduction in any medium, provided the original work is properly cited. 
cleft where the DNA substrate fits with the major groove facing the small domain and the minor groove facing the large domain. All specific DNA-protein interactions are at the small domain - major groove interface $[12,13]$. Eukaryotic C5-MTases are larger proteins but the sequence homology they share with prokaryotic C5MTases and the available biochemical data suggest that they have the same catalytic mechanism $[14,15]$.

Although the vast majority of characterized C5MTases function as monomers, there are exceptions: $\mathrm{M}$. AquI (ㄷYCGRG) and M.EcoHK31I (YGGCCR) consist of two polypeptides. The larger subunit of $\bar{M}$.AquI contains conserved motifs I - VIII and part of the variable region, whereas the smaller subunit contains the distal half of the variable region and conserved motifs IX - X $[16,17]$. In M.EcoHK31I, the larger subunit encompasses conserved motifs I - VIII, X as well as the predicted target recognition domain (TRD), and only motif IX is located in the smaller polypeptide [18]. The structural plasticity of C5-MTases is also supported by the phenomenon of protein fragment complementation observed with three enzymes: $\mathrm{N}$ - and C-terminal inactive fragments of three naturally monomeric C5MTases (M.BspRI, M.BsuRI and M.HhaI) can assemble to form active MTase if expressed in the same E. coli cell, $[19,20]$.

The goal of this work was to test whether M.SssI, which has the same specificity (CG) as the eukaryotic DNA MTases [21] and therefore has special importance as an experimental tool in the study of eukaryotic DNA methylation, is capable of fragment complementation. In higher eukaryotes, DNA methylation occurs at CG dinucleotides (CpG sites) and is associated with gene silencing [22]. Targeted DNA methylation, i.e. selective methylation of predetermined $\mathrm{CpG}$ sites in the genome is emerging as a promising technique for selective gene silencing [23-27]. The applicability of targeted methylation as a research tool or as a potential therapeutic approach critically depends on the specificity of targeting, $i$. e. on the difference of methylation between targeted and non-targeted sites. One approach to increase targeting specificity capitalized on the phenomenon of functional complementation between inactive fragments of the MTase. In the first implementation of this technique complementing $\mathrm{N}$ - and $\mathrm{C}$-terminal fragments of the HhaI MTase were genetically fused to zinc finger proteins (ZFP) engineered to recognize different nine bp sequences. When the MTase fragment-ZFP fusion proteins were expressed in the same E. coli cell, the targeted M.HhaI recognition site, which was flanked by the two closely spaced ZFP binding sites, became methylated, whereas the other M.Hhal recognition sites on the same plasmid stayed unmethylated [28]. Although this strategy is likely to require improvement to suppress the non- targeted background methylation mainly deriving from the reconstitution of the MTase in unbound state [29], it probably remains the most promising approach for achieving the specificity required for using targeted methylation as a reliable research tool [30,31]. However, of the C5-MTases shown to possess the capacity of fragment complementation, only M.HhaI can be used to target CpG sites, and even M.HhaI can methylate only a small subset of CpG sites (1 in 16, those in GCGC context). To be able to target any $\mathrm{CpG}$ site, one needs a C5MTase with CG specificity such as M.SssI.

Here, we report that M.SssI shows the phenomenon of fragment complementation, thus it is, in principle, suitable for the split fragment approach of targeted DNA methylation.

\section{Results}

\section{Construction of plasmids expressing truncated fragments of M.Sssl}

M.SssI consists of 386 amino acids and contains all conserved sequence motifs typical for C5-MTases [21] (Figure 1). There is no X-ray structure available for $\mathrm{M}$. SssI, but a computational model has been created using previously solved structures of the HhaI and HaeIII MTases [32]. According to this model, M.SssI has the same global architecture as M.HhaI and M.HaeIII, it consists of a large domain comprising conserved motifs I-VIII and X, and a small domain containing conserved motif IX and the TRD. The function of several residues predicted by sequence homology and the computational model was tested by mutational analysis [8].

To test complementation between split fragments of M.SssI in vivo, the gene segments encoding N-terminal fragments were cloned in pBAD24 $\left(\mathrm{Ap}^{\mathrm{R}}\right)$, whereas the gene segments coding for $\mathrm{C}$-terminal fragments were cloned in the compatible plasmid vector pOK-BAD $\left(\mathrm{Kn}^{\mathrm{R}}\right)$. In both vectors transcription of the target gene is under the control of the $\operatorname{araBAD}$ promoter and the $\mathrm{AraC}$ protein, expression can be induced by arabinose and repressed by glucose [34,35]. Placing the genes of the $\mathrm{N}$ - and C-terminal fragments under the same transcriptional control allowed coordinated expression of the two fragments. For future applications, almost all fragments were designed to carry $\mathrm{N}$ - or C-terminal $6 x$ Histag. Because one of our aims was to explore possibilities of chemical coupling of the MTase to triplex forming oligonucleotides [36], the C-terminal His-tag was engineered to carry a cysteine as the last amino acid (Table 1).

\section{Functional complementation between inactive fragments of M.Sssl in vivo}

First two plasmids (pB-Sss[1-189] and pBNH-Sss[1304]) expressing N-terminal, and four plasmids (pOB- 
Sss[191-386], pOB-Sss[241-386], pOB-Sss[279-386], pOB-Sss[304-386]) expressing C-terminal polypeptides were constructed. (Figure 2 and Table 1, the numbers in square brackets indicate the first and the last amino acid of the M.SssI fragment encoded by the plasmid.) Some of the break points were selected to approximately match the ends or the beginnings of fragments of the other C5-MTases, for which the phenomenon of fragment complementation has been demonstrated [19] [20]. Plasmids coding for $\mathrm{N}$ - or C-terminal fragments were introduced individually or pairwise into E.coli DH10B. The $\operatorname{rec} A$ host was used in these experiments to minimize the possibility of recombination between homologous segments of the two plasmids. Transformants were grown in the absence or presence of the inducer arabinose overnight. Methylation status of plasmid DNA isolated from the cultures was tested by digestion with the GCGC-specific restriction endonuclease Hin6I whose activity is blocked by M.SssI-specific methylation. The vectors pBAD24 and pOK-BAD contain 32 and 21 Hin6I sites, respectively.

As expected, all plasmids purified from singletransformants were fully digestible with Hin6I, indicating that the truncated fragments were inactive. Similarly, the plasmids purified from double-transformants expressing the fragment pairs [1-189]+[191-386] or [1-304] + [304-386] were completely digestible. However, plasmid preparations purified from the arabinose-induced cultures of the double-transformants containing pBNHSss[1-304] plus pOB-Sss[191-386] or pOB-Sss[241-386] were partially protected against Hin6I digestion (Figure 3). This suggested that the $\mathrm{N}$-terminal fragment [1-304] could assemble with the [191-386] or the [241-386] C-terminal fragment to reconstitute the active MTase. The [1-304] + [279-386] combination gave barely detectable protection (not shown). No protection was 
Table 1 Plasmids encoding $\mathbf{N}$ - or C-terminal fragments of M.Sssl

\begin{tabular}{|c|c|c|c|}
\hline Plasmid & Res. & $\mathrm{N}$-terminal extension ${ }^{1}$ & C-terminal extension ${ }^{1}$ \\
\hline pB-Sss[1-189] & Ap & & $\mathrm{El}$ \\
\hline pBNH-Sss[1-176] & Ap & $\mathrm{MVPGMH}_{6} \mathrm{LEC}$ & KLGCFGG \\
\hline pBNH-Sss[1-222] & Ap & $\mathrm{MVPGMH}_{6} \mathrm{LEC}$ & ASLAVLADERRFSA \\
\hline pBNH-Sss[1-239] & Ap & $\mathrm{MVPGMH}_{6} \mathrm{LEC}$ & SCFGG \\
\hline pBNH-Sss[1-248] & Ap & $\mathrm{MVPGMH}_{6} \mathrm{LEC}$ & LAVLADERRFSA \\
\hline pBNH-Sss[1-257] & Ap & $\mathrm{MVPGMH}_{6} \mathrm{LEC}$ & EAWLFWRMREDFQPDTD \\
\hline pBNH-Sss[1-304] & Ap & $\mathrm{MVPGMH}_{6} \mathrm{LEC}$ & \\
\hline pBNH-Sss[1-317] & Ap & $\mathrm{MVPGMH}_{6} \mathrm{LEC}$ & KLGCFGG \\
\hline pBNH-Sss[1-318] & Ap & $\mathrm{MVPGMH}_{6} \mathrm{LEC}$ & KLGCFGG \\
\hline pBNH-Sss[1-324] & Ap & $\mathrm{MVPGMH}_{6} \mathrm{LEC}$ & LGCFGG \\
\hline pBNH-Sss[1-356] & Ap & $\mathrm{MVPGMH}_{6} \mathrm{LEC}$ & KLGCFGG \\
\hline pB6ZB-Sss[1-239] & Ap & 6-ZFP-B zinc finger domain & SCFGG \\
\hline pB6ZB-Sss[1-304] & Ap & 6-ZFP-B zinc finger domain & \\
\hline pOB-Sss[191-386] & $\mathrm{Kn}$ & MVP & $\mathrm{SH}_{6} \mathrm{C}$ \\
\hline pOB-Sss[231-386] & $\mathrm{Kn}$ & MVP & $\mathrm{SH}_{6} \mathrm{C}$ \\
\hline pOB-Sss[240-386] & $\mathrm{Kn}$ & MV & $\mathrm{SH}_{6} \mathrm{C}$ \\
\hline pOB-Sss[241-386] & Kn & M & $\mathrm{SH}_{6} \mathrm{C}$ \\
\hline pOB-Sss[242-386] & Kn & MVQ & $\mathrm{SH}_{6} \mathrm{C}$ \\
\hline pOB-Sss[246-386] & $\mathrm{Kn}$ & MV & $\mathrm{SH}_{6} \mathrm{C}$ \\
\hline pOB-Sss[250-386] & $\mathrm{Kn}$ & $\mathrm{MVH}$ & $\mathrm{SH}_{6} \mathrm{C}$ \\
\hline pOB-Sss[254-386] & Kn & MVP & $\mathrm{SH}_{6} \mathrm{C}$ \\
\hline pOB-Sss[261-386] & Kn & MV & $\mathrm{SH}_{6} \mathrm{C}$ \\
\hline pOB-Sss[279-386] & Kn & M & $\mathrm{SH}_{6} \mathrm{C}$ \\
\hline pOB-Sss[304-386] & $\mathrm{Kn}$ & M & $\mathrm{SH}_{6} \mathrm{C}$ \\
\hline pOB-Sss[316-386] & Kn & MVP & $\mathrm{SH}_{6} \mathrm{C}$ \\
\hline pOB-Sss[321-386] & $\mathrm{Kn}$ & MVP & $\mathrm{SH}_{6} \mathrm{C}$ \\
\hline pSss[191-386] del ${ }^{2}$ & $\mathrm{Kn}$ & MVP & $\mathrm{SH}_{6} \mathrm{C}$ \\
\hline pSss[241-386]del ${ }^{2}$ & $\mathrm{Kn}$ & M & $\mathrm{SH}_{6} \mathrm{C}$ \\
\hline pOB-Sss[191-386]SS & $\mathrm{Kn}$ & & $\mathrm{SH}_{6} \mathrm{C}$ \\
\hline pOB-Sss[241-386]-6ZA & $\mathrm{Kn}$ & M & 6-ZFP-A zinc finger domain \\
\hline pOB-Sss[261-386]-6ZA & Kn & MV & 6-ZFP-A zinc finger domain \\
\hline pBS-CAL75 & Ap & & \\
\hline pBS-Sss6ZA & Ap & & 6-ZFP-A zinc finger domain \\
\hline
\end{tabular}

Numbers in square brackets specify the M.Sssl fragment expressed by the plasmid.

${ }^{1} \mathrm{~N}$ - and C-terminal extensions were added on purpose (His tag) or are byproducts of the cloning procedure. ${ }^{2}$ Plasmid lacking the $P_{B A D}$ promoter.

observed with plasmids purified from uninduced cultures and complementation was more efficient at $30{ }^{\circ} \mathrm{C}$ than at $37^{\circ} \mathrm{C}$.

Since the truncated genes of the complementing Nand C-terminal polypeptides contained overlapping segments, it was important to exclude the possibility that MTase activity arose by reconstitution of the intact MTase gene via homologous recombination. To test this, the $\operatorname{ara} B A D$ promoter and part of the $\operatorname{araC}$ gene was deleted from pOB-Sss[191-386] and pOB-Sss[241-386] leaving the [191-386] and [241-386] coding sequence intact. The resulting plasmids (pSss[191-386]del and pSss[241-386]del) did not complement pBNH-Sss[1304] (not shown) indicating that for restoration of MTase activity synthesis of the C-terminal peptide was required, and a recombination mechanism can be excluded.

To determine the optimal C-terminal fragment length for complementation, nested deletions were generated by exonuclease III digestion starting from the $\mathrm{N}$ terminus of the [191-386] fragment as described in Methods. The obtained plasmids encoding the truncated polypeptides [231-386], [240-386], [242-386], [246-386], 


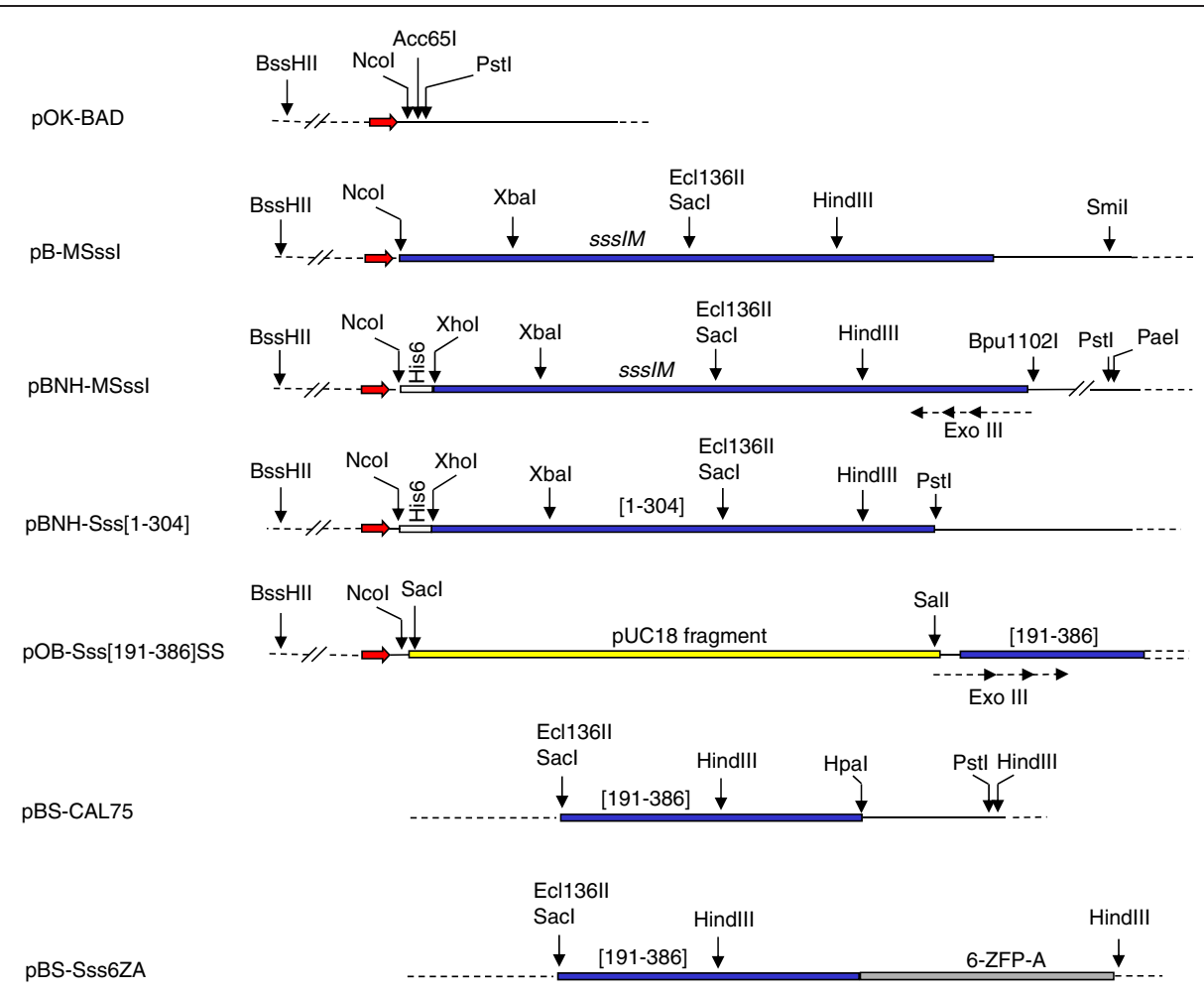

Figure 2 Schematic map of the relevant regions of some plasmids used in this study. Blue horizontal bar, sss/M gene coding sequence; gray horizontal bar, 6-ZFP-A zinc finger protein gene; red arrow, araBAD promoter. Restriction sites used in plasmid constructions are shown. Maps are not drawn to scale.

[250-386], [254-386], [261-386], [316-386] and [321-386] are listed in Table 1. When tested in combination with pBNH-Sss[1-304], some protection against Hin6I digestion was detectable for all fragments having overlaps with fragment [1-304]. The most efficient methylation was observed with fragments starting at 241, 242 and 246 (Figure 4A). These residues are located between conserved motif VIII and the assumed TRD of M.SssI (Figure 1).

In a reciprocal approach, nested deletions were introduced from the 3'-end of the M.SssI gene. Plasmids expressing the following polypeptides were isolated: [1-176], [1-222], [1-239], [1-248], [1-257], [1-317], [1-318], [1-324] and [1-356] (Table 1 and Figure 1). Because in these plasmids the truncated gene was fused to vector sequence, translation termination is determined by the next in-frame stop codon downstream of the PaeI site (Figure 2), and the fragments carry short C-terminal appendages of foreign sequence (Table 1). These plasmids were tested in combination with pOB-Sss[241-386] and pOB-Sss[242-386], two of the plasmids that most efficiently complemented the N-terminal polypeptide [1-304]. The two shortest fragments $([1-176]$ and [1-222]) did not complement [241-386] (Figure 4B). Complementation by fragment
[1-239] was rather efficient, whereas only poor methylation activity was observed with fragments [1-248] and [1-257]. The low complementation capacity of the latter two fragments is probably due to the relatively long foreign sequence at their C-terminus (Table 1). All longer fragments were active in the complementation test and resulted in comparable levels of methylation (Figure 4B). Similar results were obtained when [242-386] was used as C-terminal fragment although in this combination fragments [1-248/257] appeared more active than with [241-386] (Figure $4 \mathrm{C}$ vs. $4 \mathrm{~B})$. The complementation observed with the $[1-239]+[241 /$ 242-386] pairs is noteworthy because in these cases there is no overlap between the fragments.

It has been observed in several systems that sequence overlap between the fragment pairs was beneficial or even essential for complementation [20,37,38]. To test this requirement for M.SssI in more detail, a series of Cterminal fragments were tested in combination with [1239]. Fragments [240/241/242-386] were highly active in pair with [1-239], whereas the few residues shorter [246-386] fragment was already ineffective (Figure 4D). It was shown before that the $[1-189]+[191-386]$ and the $[1-304]+[304-386]$ combinations were inactive (see above). Taking into account that fragments [1-304] and 


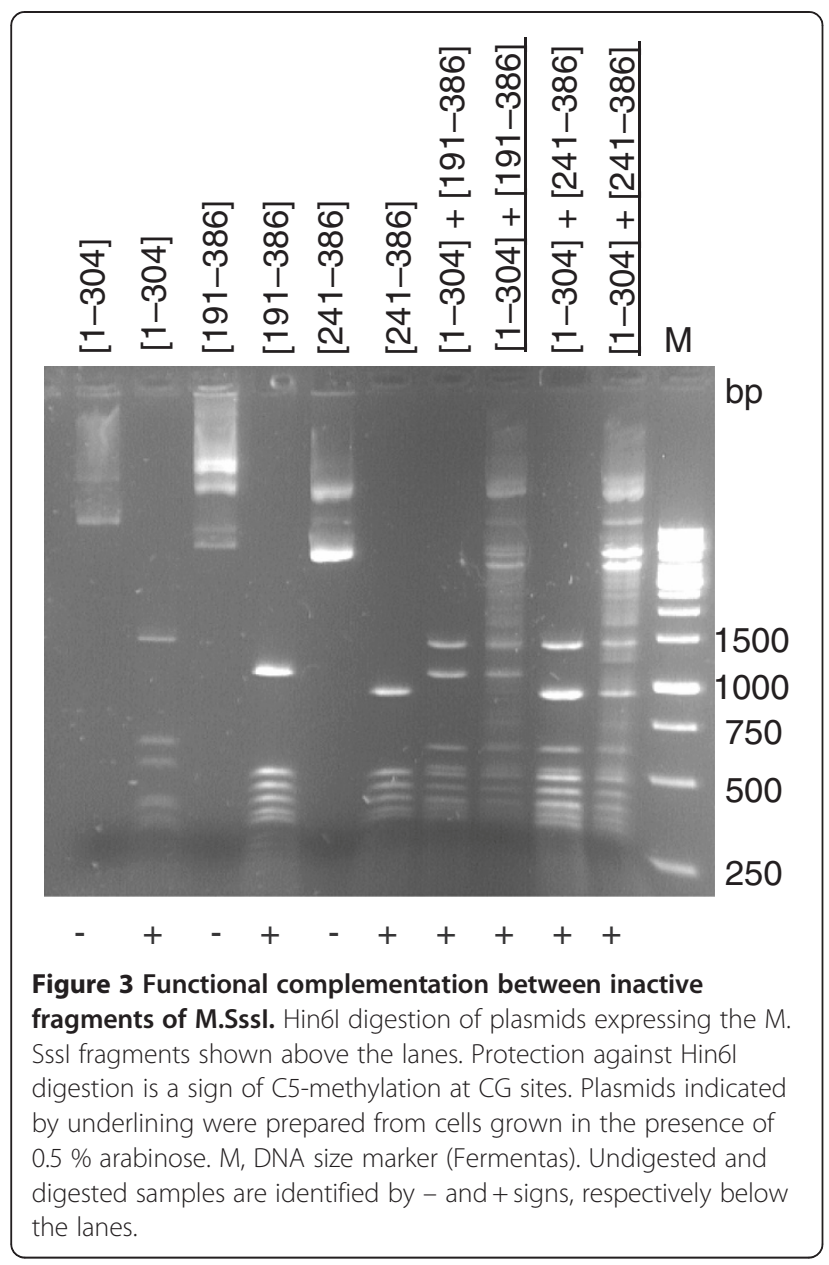

[191-386] were active in combination with fragment(s), which they overlapped, these observations show that the requirement for overlap depends on the location of the break. If the bisection point is in the proximal half of the variable region, no overlap is required, and even the loss of a few amino acids can be compatible with MTase activity. In contrast, if the bisection point is in regions less optimal for fitting the two polypeptides together, a short overlap between the fragment pairs appears to be necessary for the formation of the active complex.

To test how M.SssI tolerates splits in more distal parts of the variable region, plasmids producing different $\mathrm{N}$ terminal fragments were tested in combination with pOB-Sss[279-386] and pOB-Sss[304-386]. Methylation of the plasmid pairs expressing fragments [1-304/317/ $324]+[279-386]$ was hardly detectable, and no methylation was detected with the $[1-317 / 318 / 324]+[304-386]$ combinations (not shown). These results and the previous observation that the $[1-304]+[304-386]$ pair was inactive (see above) suggest that for efficient complementation the small domain must be intact. A summary of the complementation properties of M.SssI fragments generated and studied in this work is in Figure 5.
To compare M.SssI activities in vivo between representatives of the best complementing fragment pairs and intact M.SssI, extracts were prepared from arabinose induced cells and MTase activity was determined using radioactively labeled AdoMet as described in Methods. The following MTase activities (derived from three independent experiments and expressed in $\mathrm{cpm} / \mu \mathrm{g}$ extract protein) were obtained (with the enzyme in parentheses): $1016 \pm 369$ (full-length), $16 \pm 0$ ([1-304]), 53 \pm 14 ([1$304]+[242-386])$ and $65 \pm 8([1-304]+[246-386])$. Assuming similar levels of expression, these values suggest that the activities of even the best complementing pairs is below 5 per cent of that of the intact enzyme.

In the envisaged approach of targeted methylation, targeting is achieved by zinc finger proteins fused to the MTase halves [28]. Statistically, a 16 bp sequence occurs once in the human genome. Thus, for the intended specificity, a 18 bp zinc finger binding site should be sufficiently long as has been demonstrated [39]. Therefore, for testing whether fusion of the M.SssI fragments to zinc finger proteins could impair their complementation ability, we chose six-finger proteins that recognize $18 \mathrm{bp}$ sequences. Two $\mathrm{N}$ - and two C-terminal M.SssI fragments ([1-239], [1-304], [241-386] and [261-386]) were genetically fused to the zinc finger domains 6-ZFP$A$ and 6-ZFP-B described in [40]. The 6-ZFP-B domain was fused to the amino end of the $\mathrm{N}$-terminal fragments, whereas the 6-ZFP-A domain was fused to the carboxy end of the C-terminal fragments to yield pB6ZB-Sss[1239], pB6ZB-Sss[1-304], pOB-Sss[241-386]-6ZA and pOB-Sss[261-386]-6ZA (Table 1). Fusions to ZFPs slightly decreased complementation capacity in all tested cases (Figure 6 vs. Figure 4), but with the two better combinations substantial activity remained and even for the fragment pair displaying the lowest activity ([1$304]+[261-386]$, Figure 4A) appending the ZFPs did not fully abolish MTase activity (Figure 6).

\section{Discussion}

In this study we identified $\mathrm{N}$ - and C-terminal fragments of the SssI MTase, which can assemble with a counterpart fragment to form active MTase when produced in the same E. coli cell. All available evidence supports the conclusion that the observed phenomenon is indeed peptide complementation and not the result of DNA recombination restoring the intact MTase gene. Firstly, deletion of the promoter of two C-terminal fragments abolished complementation (see above). Secondly, plasmid pairs with longer overlaps between the 5'- and 3' gene segments were not necessarily more efficient in complementation than fragments with shorter overlaps (Figure 4A). Thirdly, in the experiment in which the [191-386] fragment was shortened from the $\mathrm{N}$-terminus by exonuclease III digestion, many of the randomly 


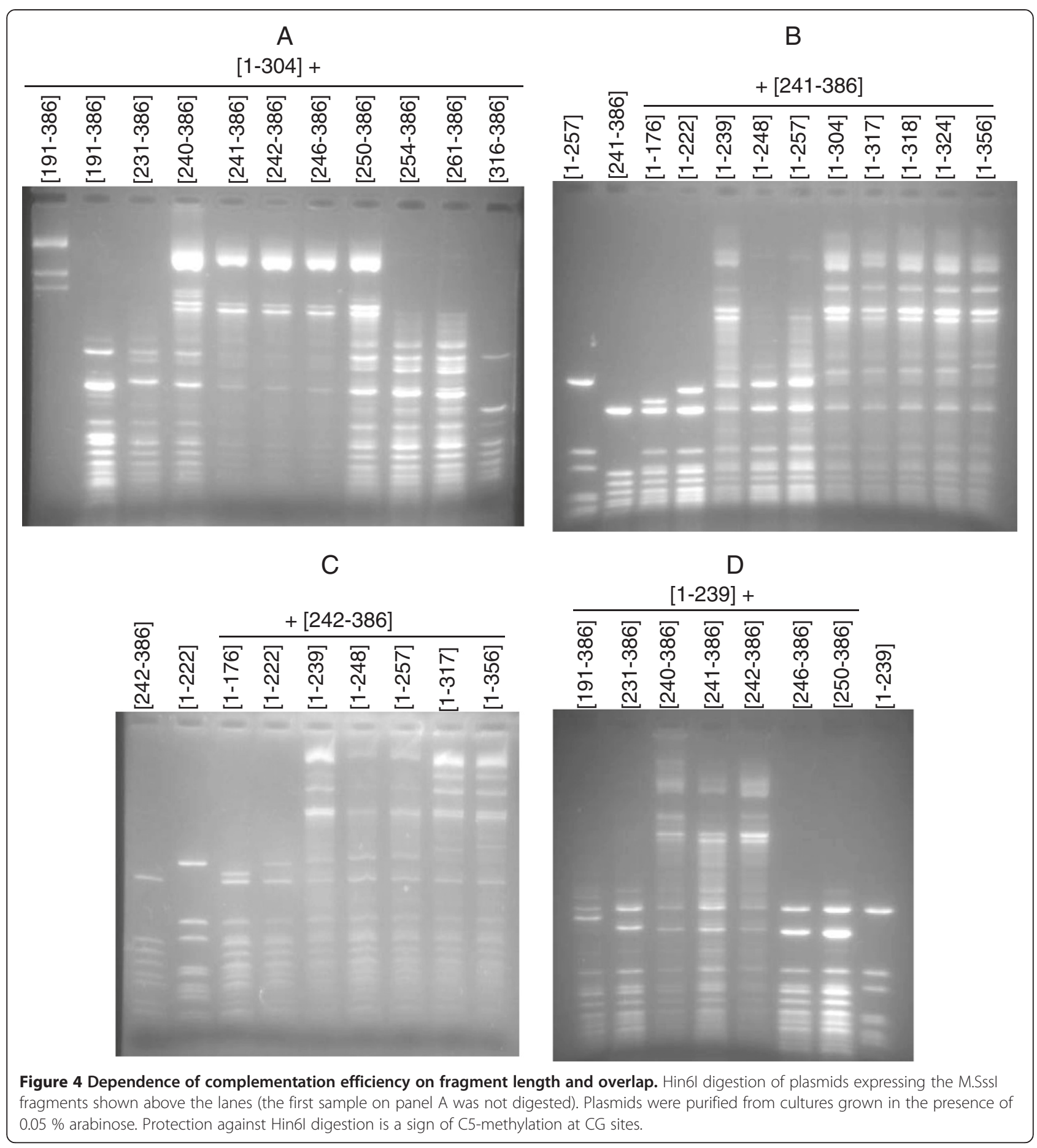

picked clones contained M.SssI gene segments with substantial overlap with the gene of the [1-304] fragment but fused to the ATG initiation codon in incorrect reading frame. When these plasmids were co-transformed with $\mathrm{pBNH}-\mathrm{Sss}[1-304]$, methylation was not detectable, confirming that M.SssI activity required synthesis of the C-terminal peptide (not shown). Finally, complementation observed between some plasmids containing non- overlapping gene segments such as $[1-239]+[241 / 242$ 386] (Figures 4D) also argues against the recombination model.

Although a large number of fragment pairs resulted in detectable M.SssI activity, the most efficient methylation was observed with fragment combinations involving $\mathrm{C}$ terminal fragments starting between conserved motif VIII and the assumed TRD, with a sharp optimum 


\begin{tabular}{|c|c|c|c|c|c|c|c|c|c|c|c|c|c|}
\hline & 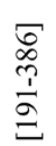 & $\begin{array}{l}\bar{\varpi} \\
\infty \\
\stackrel{1}{1} \\
\vec{\jmath}\end{array}$ & 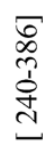 & 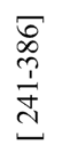 & 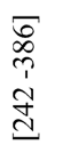 & 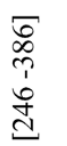 & $\begin{array}{l}\bar{\varnothing} \\
\infty \\
\stackrel{0}{1} \\
\stackrel{d}{d} \\
\stackrel{d}{d}\end{array}$ & 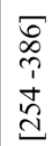 & 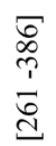 & 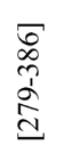 & $\begin{array}{l}0 \\
0 \\
\infty \\
0 \\
0 \\
0\end{array}$ & $\begin{array}{l}\text { D } \\
\infty \\
\text { bे } \\
\underline{n}\end{array}$ & 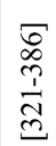 \\
\hline$[1-176]$ & & & & - & - & & & & & & & & \\
\hline [1-189] & - & & & & & & & & & & & & \\
\hline$[1-222]$ & & & & - & - & & & & & & & & \\
\hline$[1-239]$ & \pm & \pm & ++ & ++ & ++ & - & - & & & & & & \\
\hline$[1-248]$ & & & & \pm & ++ & & & & & & & & \\
\hline$[1-257]$ & & & & + & ++ & & & & & & & & \\
\hline [1-304] & + & + & ++ & +++ & +++ & +++ & ++ & + & + & \pm & - & - & - \\
\hline$[1-317]$ & & & & ++ & ++ & & & & & \pm & - & & \\
\hline$[1-318]$ & & & & ++ & ++ & & & & & & - & & \\
\hline$[1-324]$ & & & & ++ & ++ & & & & & \pm & - & & \\
\hline$[1-356]$ & & & & ++ & ++ & & & & & & & & \\
\hline
\end{tabular}

Figure $\mathbf{5}$ In vivo complementation between M.Sssl fragments. Some of the fragments carry N- or C-terminal appendages (see Table 1). The approximate level of plasmid methylation as determined by restriction protection assay is indicated by + signs, and the lack of methylation by - sign. Boxes for untested combinations were left empty.

between residues 241 and 246 (Figures 1 and 5). In lack of an X-ray structure it is difficult to rationalize these results but the location of split sites in the region connecting the predicted large and small domains [32] would be consistent with the general principle that fragment complementation is most efficient between folded units [37]. The flexible random coil conformation predicted for the segment Glu241 - Ile262 [32] could also be an advantage for fitting the two subunits together. It

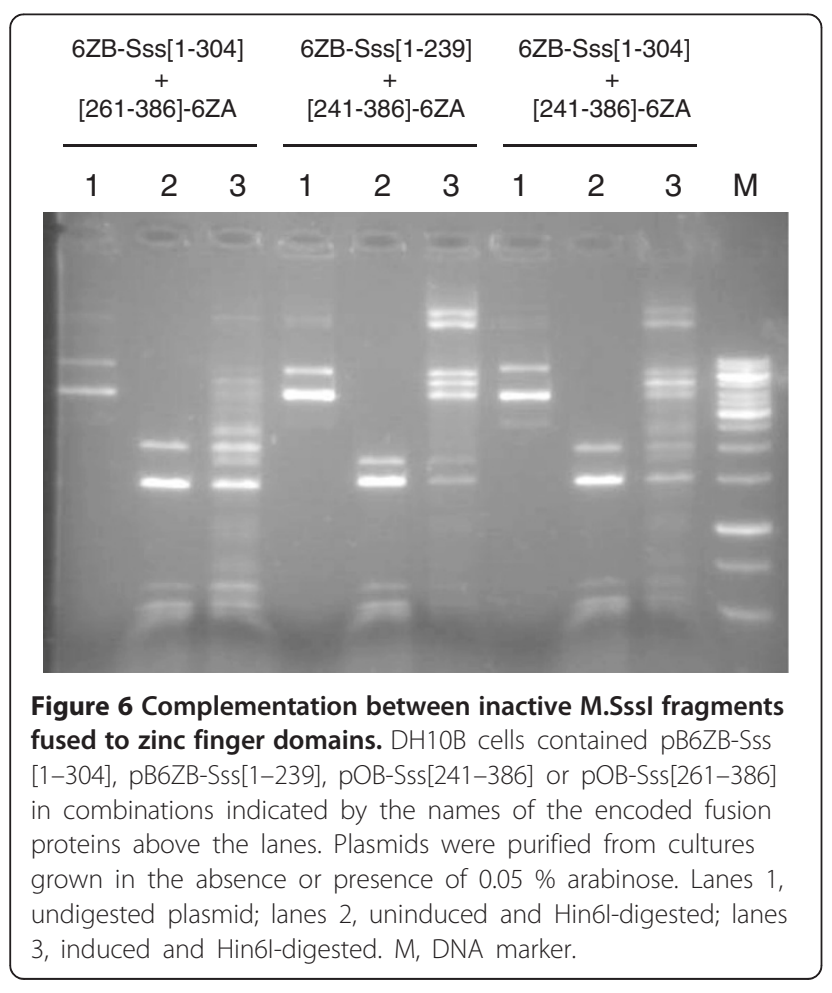

is noteworthy that the beginning (K210) of the C-terminal M.HhaI fragment producing complementation was in the same region, between motif VIII and the TRD [20].

Evaluation of the phenotypes of the N-terminal fragments generated by exonuclease III digestion is less straightforward because the extraneous C-terminal peptides appended by the cloning procedure (Table 1) are likely to interfere with assembly of the subunits. In contrast to the C-terminal fragments, the complementation ability of the $\mathrm{N}$-terminal fragments did not show a clear optimum. All overlapping fragments, which did not have longer extraneous $\mathrm{C}$-terminal peptide could complement fragments [241-386] and [242-386] and the complementation capacity of these fragments appeared to be similar (Figures $4 \mathrm{~B}$ and $\mathrm{C}$ ).

This work was motivated by the desire to develop M. SssI into a programmable DNA MTase suitable to methylate unique $\mathrm{CpG}$ sites in the genome. Here, as a first step toward this goal, the ability of M.SssI for fragment complementation has been demonstrated and fragment combinations displaying different levels of MTase activity in vivo have been identified. These results open the possibility for developing a system for targetable CpG methylation using the split fragment approach, which will involve construction of fusions between fragments of M.SssI and appropriately designed ZFP targeting domains. The split fragment approach is in many ways reminiscent of the technique using ZFP-FokI chimeric nucleases for targeted DNA cleavage [41]. Low activity of the heterodimers can be an advantage for increasing targeting specificity especially if it derives from the low affinity between the MTase halves [31]. Ideally, the split MTase fragments should not be able to 
assemble in free state at cellular concentrations but should form active heterodimers due to increased local concentration when co-localized to the target site via the ZFP domains. The best strategy to create a site specific MTase could be to start with fragments, which cannot associate into active MTase as has been suggested [29]. The level of targeting specificity required for methylation of unique sites in the genome will probably need extensive optimization of different parameters governing targetable methylation specificity, such as affinity to target and non-target DNA, affinity between the MTase subunits, distance between the ZFP binding site and the targeted CG, etc.. The large number of complementing fragment combinations representing a wide range of MTase activity will be a rich source of starting material to engineer ZFP-M.SssI fusions for programmable DNA methylation.

\section{Conclusions}

The CG-specific M.SssI, similarly to three other naturally monomeric C5-MTases of different specificity, shows the phenomenon of fragment complementation in vivo in E. coli. Fusion of the split fragments to six unit zinc finger domains only slightly decreases the efficiency of complementation. These observations offer the possibility to develop M.SssI into a programmable DNA methyltransferase of high specificity that can be useful in the study of DNA methylation in higher eukaryotes.

\section{Methods}

\section{Strains and growth conditions}

The Escherichia coli strain $\mathrm{DH} 10 \mathrm{~B} \mathrm{~F}^{-}$endA1 recA1 galE15 galK16 nupG rpsL $\triangle$ lacX74 80dlacZLM15 araD139 $\Delta$ (ara leu) 7697 mcrA $\Delta$ (mrr-hsdRMS-mcrBC) relA1 spoT1 $\lambda^{-}$[42] was used as general cloning host and CJ236 FA(HindIII)::cat $\left(\mathrm{Tra}^{+} \mathrm{Pil}^{+} \mathrm{Cam}^{\mathrm{R}}\right) /$ ung-1 relA1 dut-1 thi-1 spoT1 mcrA as host for site-directed mutagenesis.

Cells were grown in LB medium at 30 or $37^{\circ} \mathrm{C}$. Ampicillin (Ap) and kanamycin (Kn) were used at 100 and $50 \mu \mathrm{g} / \mathrm{ml}$, respectively. L-arabinose (Sigma) was used at $0.5-0.005 \%$ as indicated at the experiments.

\section{Plasmids}

pBAD24 is an expression vector plasmid, in which transcription of the target gene is tightly controlled by the araBAD promoter and the AraC protein [34]. pOK$\mathrm{BAD}$, a Kn-resistant derivative of pBAD24, has p15A replicon, thus is compatible with ColE1-based plasmids such as pBAD24 [35].

All variants of the sssIM gene described in this paper are derived from the allele in the plasmid pCAL7 obtained from New England Biolabs. The sssIM allele in pCAL7, considered as the WT allele for this work, carries mutations that change four TGA stop codons, which normally specify Trp in the native host Spiroplasma sp., to TGG (W. Jack, personal communication). The pBAD24-based plasmids pB-MSssI and pBNHMSssI encode the WT and an N-terminally His-tagged M.SssI variant, respectively (Figure 2).

Plasmids encoding N-terminal M.SssI fragments are based on the vector pBAD24, and have names starting with $\mathrm{pB}$, whereas plasmids that encode $\mathrm{C}$-terminal $\mathrm{M}$. SssI fragments, are based on the vector pOK-BAD and carry names starting with $\mathrm{pOB}$ - (Table 1 ).

Plasmid pB-Sss[1-189] was constructed by deleting the Ecl136II - Smil fragment of pB-MSssI (Figure 2). Ecl136II cuts after the codons of the conserved $\mathrm{E}_{186} \mathrm{NV}$ motif and Smil after the stop codon. This deletion created a reading frame ending two triplets downstream of the $\mathrm{Gly}_{189}$ codon. Thus, the MTase fragment encoded by pB-Sss[1-189] carries a two amino acid C-terminal appendage ENVGEI (underlined).

pBNH-Sss[1-304] is a derivative of pBNH-MSssI, and encodes the [1-304] fragment of M.SssI. To construct pBNH-Sss[1-304], the M.SssI gene segment corresponding to the peptide $A_{190}-V_{304}$ was PCR-amplified using the primers AK183 and AK184. AK183 contains the Ecl136II (SacI) site located in the middle of the SssI gene. AK184 introduced a stop codon and a PstI site after the triplet corresponding to $\mathrm{Val}_{304}$ (Table 2). The PCR-product was T/A-cloned in pTZ57R/T (Fermentas), then excised by SacI-PstI double digestion and transferred into SacI-PstI digested pBNH-MSssI to yield pBNH-Sss[1-304] (Figure 2).

Plasmids pBNH-Sss[1-176], pBNH-Sss[1-222], pBNH-Sss[1-239], pBNH-Sss[1-248], pBNH-Sss[1-257], pBNH-Sss[1-317], pBNH-Sss[1-318], pBNH-Sss[1-324] and $\mathrm{pBNH}-\mathrm{Sss}[1-356]$ are deletion derivatives of $\mathrm{pBNH}-$ MSssI and were generated by exonuclease III digestion. pBNH-MSssI was digested with Bpu1102I and PaeI, which have single sites in the plasmid downstream of the M.SssI gene (Figure 2). Unidirectional deletions starting from the Bpu1102I end (5'-overhang) were made by exonuclease III [43]. The 3'-overhang of the PaeI end protected the downstream sequences. The M.SssI fragments expressed from the plasmids obtained by exonuclease digestion carry short $\mathrm{C}$-terminal appendages (Table 1) determined by the fused vector sequence downstream of the PaeI site.

Plasmids pOB-Sss[191-386], pOB-Sss[241-386], pOBSss[279-386] and pOB-Sss[304-386] are based on the expression vector pOK-BAD and encode C-terminal M. SssI fragments. They were constructed either by direct fragment transfer or PCR amplification from a plasmid expressing a C-terminally His-tagged M.SssI variant. This variant and the derived C-terminal polypeptides used in this work carry a cysteine at the end of the His- 
Table 2 Deoxyoligonucleotides used in this work

\begin{tabular}{|c|c|c|c|}
\hline Name & Sequence & Properties $^{1}$ & Use \\
\hline AK183 & GAGCTCTTCTTCACAAGAAGA & $\begin{array}{l}\text { M.Sssl gene sense strand, } \\
\text { positions } 566-586 \text {. Starts } \\
\text { with a Sacl site }\end{array}$ & $\begin{array}{l}\text { Forward primer for } \\
\text { [190-304] }\end{array}$ \\
\hline AK184 & GCTGCAGTTAAACATAACCTTCTGAATT & $\begin{array}{l}\text { M.Sssl gene anti-sense } \\
\text { strand, positions } 912-895, \\
\text { stop codon and Pstl site added }\end{array}$ & $\begin{array}{l}\text { Reverse primer for } \\
\text { [1-304] }\end{array}$ \\
\hline AK185 & 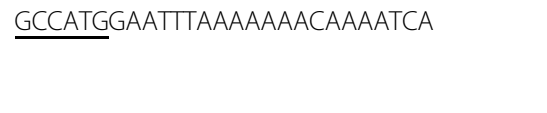 & $\begin{array}{l}\text { M.Sssl gene sense strand, } \\
\text { positions 835-855, Ncol site } \\
\text { added }\end{array}$ & $\begin{array}{l}\text { Forward primer for } \\
\text { [279-386] }\end{array}$ \\
\hline AK186 & $\underline{\text { GCTGCAGTTAGCAGTGATGGTG }}$ & $\begin{array}{l}\text { M.Sssl gene anti-sense } \\
\text { strand, stop codon and Pstl } \\
\text { site added }\end{array}$ & $\begin{array}{l}\text { Reverse primer for } \\
\text { His }_{6} \text {-Cys-tagged } \\
\text { C-terminal fragments }\end{array}$ \\
\hline AK188 & GCCATGGTTTATGATCCTGAATTTACA & $\begin{array}{l}\text { M.Sssl gene sense strand, } \\
\text { positions 910-930, Ncol site } \\
\text { added }\end{array}$ & $\begin{array}{l}\text { Forward primer for } \\
\text { [304-386] }\end{array}$ \\
\hline AK189 & GCCATGGAATTTGTTGAACTACCAAAG & $\begin{array}{l}\text { M.Sssl gene sense strand, } \\
\text { positions } 721-741 \text {, Ncol site } \\
\text { added }\end{array}$ & $\begin{array}{l}\text { Forward primer for } \\
\text { [241-386] }\end{array}$ \\
\hline AK258 & GGCACGCTGACTAAAGGATTTACCGCACTCGGG & $\begin{array}{l}\text { 6-ZFP-A gene antisense } \\
\text { strand }\end{array}$ & $\begin{array}{l}\text { Mutagenic primer } \\
\text { for elimination of a } \\
\text { Hindlll site }\end{array}$ \\
\hline AK259 & GCAGCTGTTCTCCAAAGAAGAAGAGAAAAGT & & $\begin{array}{l}\text { Forward primer for } \\
\text { amplification of } \\
\text { the 6-ZFP-A gene }\end{array}$ \\
\hline AK260 & GCTGCAGTTAACCCAGCTCGCCG & & $\begin{array}{l}\text { Reverse primer for } \\
\text { amplification of } \\
\text { the 6-ZFP-A gene }\end{array}$ \\
\hline
\end{tabular}

Sequences are written in $5^{\prime}$ to $3^{\prime}$ direction. Sequences, which do not correspond to the template DNA, are underlined. ${ }^{1}$ Nucleotide positions are calculated relative to the first nucleotide of the ATG start codon of the WT M.Sssl gene.

tag: KIGGSHHHHHHC. (The C-terminal cysteine, not relevant to the present study, was added for future applications.) Plasmid pOB-Sss[191-386] was constructed by cloning the Ecl136II-PstI fragment encoding the Histagged [191-386] polypeptide between the filled-in Acc65I site and the PstI site in the pOK-BAD polylinker. The variant encoded by pOB-Sss[191-386] has the following N-terminal sequence MVPL $_{191}$ LHKK. The underlined three amino acid extension is encoded by the pOK-BAD polylinker. To construct plasmids expressing the [241-386], [279-386] and [304-386] polypeptides, the respective gene segments were PCR-amplified using AK189, AK185 or AK188 as forward primer and AK186 as reverse primer (Table 2). In pOK-BAD the NcoI site overlaps the translational start codon. To facilitate cloning of the synthesized fragments in pOK-BAD, the forward primers were designed to carry a 5 '-extension with an NcoI site. The amplified fragments were inserted between the NcoI and PstI sites of pOK-BAD.

Plasmids pSss[191-386]del and pSss[241-386]del were made by deleting the BssHII-NcoI fragment of pOB-Sss [191-386] and pOB-Sss[241-386], respectively. BssHII cuts in the middle of the $\operatorname{araC}$ gene and the NcoI site contains the ATG start codon of the truncated MTase genes (Figure 2). Thus, this deletion removes part of the $\operatorname{ara} C$ gene along with the $\operatorname{araBAD}$ promoter and translational initiation signals for the [191-386] and [241-386] polypeptides.

Plasmids pOB-Sss[231-386], pOB-Sss[240-386], pOBSss[242-386], pOB-Sss[246-386], pOB-Sss[250-386], pOB-Sss[254-386], pOB-Sss[261-386], pOB-Sss[316-386] and pOB-Sss[321-386] are deletion derivatives of pOB-Sss[191-386] and were obtained by exonuclease III digestion. To have appropriate restriction sites for unidirectional exonuclease III treatment, pUC18 linearized with KpnI was inserted into the unique KpnI site of pOB-Sss[191-386] located in the gene section coding for the N-terminal MVP appendage (see above) i.e. between the start codon and the gene segment encoding the [191-386] fragment. A plasmid, in which the orientation of pUC18 was such that the unique SacI site was upstream (towards the translational start codon), whereas the unique Sall site was downstream, was chosen for further work. Part of the inserted pUC18, which was not needed for controlling the exonuclease III digestion, was deleted by ApaLI digestion and circularization of the large fragment to yield pOB-Sss [191-386]SS (Figure 2). To generate random 5'-deletions in the gene of the [191-386] fragment, pOB-Sss[191386]SS was cut with SalI (5'-overhang) and SacI (3'- 
overhang) and treated with exonuclease III as described above. The Sall end allowed exonuclease III digestion to start whereas the SacI end protected upstream sequences, such as the $\mathrm{P}_{\mathrm{BAD}}$ promoter and the initiation codon. As byproducts of the cloning steps, some of the M.SssI fragments encoded by these plasmids carry short $\mathrm{N}$-terminal extensions (Table 1).

The plasmids pcDNA3.1mnhk-up1 and pcDNA3.1mnhkup2 encode the zinc finger proteins 6-ZFP-A and 6-ZFP-B, respectively [40], and were kindly provided by $\mathrm{M}$. Rots. To construct fusions between the N-terminal M.SssI fragments [1-304], [1-239] and 6-ZFP-B, the 6-ZFP-B coding region was transferred, on an NcoI-XhoI fragment, from pcDNA3.1mnhk-up2, into pBNH-Sss[1-304] and pBNHSss[1-239] to yield pB6ZB-Sss[1-304] and pB6ZB-Sss[1239], respectively. For fusions to C-terminal M.SssI fragments, the HindIII site in the 6-ZFP-A zinc finger gene was eliminated by site directed mutagenesis using the mutagenic primer AK258. The resulting 6-ZFP-A gene carrying the silent mutation was PCR-amplified using primers AK259 and AK260 containing PvuII and PstI sites as 5'extensions, respectively (Table 2 ). The PCR product was $\mathrm{T} /$ A-cloned in pTZ57R/T, then excised by PvuII - PstI double-digestion and cloned between the HpaI and PstI sites of pBS-CAL75, a plasmid containing the 3'-half of the sssIM gene with an engineered HpaI site overlapping the M.SssI stop codon (Figure 2). Ligation of the Pvull end of the PCR fragment to the HpaI end of pBS-CAL75 creates in-frame fusion between the MTase and the 6-ZFP-A zinc finger domain (Figure 2, plasmid pBS-Sss6ZA). C-terminal fusions between the M.SssI fragments [241-386]/[261-386] and 6-ZFP-A were constructed by inserting the HindIII fragment of pBS-Sss6ZA containing the 3'-end of the M. SssI gene and the coding sequence of 6-ZFP-A (Figure 2) into the unique HindIII site located in the 3' half of the M. SssI coding sequence (Figure 2) in pOB-Sss[241-386] and pOB-Sss[261-386] to yield pOB-Sss[241-386]-6ZA and pOB-Sss[261-386]-6ZA, respectively.

Nucleotide sequences of the plasmids are available upon request.

\section{DNA techniques}

Restriction digestion, polymerase chain reaction, agarose gel electrophoresis and cloning in E. coli plasmid vectors was carried out using standard procedures [43]. Oligonucleotides were purchased from IDT or were synthesized in this institute and are listed in Table 2. Restriction endonucleases, DNA polymerase large (Klenow) fragment, Taq DNA polymerase, exonuclease III, S1 nuclease and T4 DNA ligase were purchased from Fermentas or New England Biolabs. Site-directed mutagenesis was performed by the Kunkel method [44]. Unidirectional deletions were generated by the combined use of exonuclease III, S1 nuclease, Klenow DNA polymerase and T4 DNA ligase [43]. DNA sequence was determined by an automated sequencer (ABI).

\section{Methyltransferase assay in crude extracts}

E. coli cells with plasmids encoding M.SssI fragment(s) or the full-length enzyme were grown at $37{ }^{\circ} \mathrm{C}$ to $\mathrm{OD}_{550} \sim 0.5$, then $0.1 \%$ arabinose was added and culturing was continued for $3 \mathrm{hrs}$ at $30{ }^{\circ} \mathrm{C}$. Cells sedimented from $200 \mathrm{ml}$ culture by centrifugation were resuspended in $0.2 \mathrm{ml} 50 \mathrm{mM}$ Tris- $\mathrm{HCl} \mathrm{pH} 8.0,10 \mathrm{mM}$ 2-mercaptoethanol and disrupted by sonication. After removing cell debris by centrifugation, M.SssI activity was determined in $30 \mu \mathrm{l}$ reactions containing $0.9 \mu \mathrm{g}$ lambda phage DNA (dam $\left.{ }^{-} d_{c m}^{-}\right), 50 \mathrm{mM}$ Tris- $\mathrm{HCl} \mathrm{pH} 8.5,50 \mathrm{mM}$ $\mathrm{NaCl}, 10 \mathrm{mM}$ EDTA, $5 \mathrm{mM}$ DTT, $5 \mu \mathrm{M}$ [methyl- ${ }^{3} \mathrm{H}$ ] AdoMet and $2 \mu \mathrm{l}$ cell extract. The [methyl ${ }^{3} \mathrm{H}$ ] AdoMet used in the reaction was prepared by diluting the $370 \mathrm{GBq} / \mathrm{mmol}$ preparation (NET155, New England Nuclear) to $116 \mathrm{GBq} / \mathrm{mmol}$ with unlabeled AdoMet (New England Biolabs). After incubation at $30{ }^{\circ} \mathrm{C}$ for $20 \mathrm{~min}$, reactions were stopped by $1 \% \mathrm{SDS}$, then the incorporated radioactivity was determined as described [45]. Protein concentration was determined by the Bradford reaction (Sigma). MTase activity was calculated by dividing the cpm values by the amount of total protein in $2 \mu \mathrm{l}$ cell extract (in $\mu \mathrm{g}$ ) used in the assay.

\section{Competing interests}

The authors declare that they have no competing interests.

\section{Acknowledgements}

We thank William Jack (New England Biolabs) for the plasmid pCAL7, Marianne Rots (University Medical Center Groningen) for plasmids encoding zinc finger domains, Tamás Raskó for pBS-CAL75 and for contribution to construct pBNH-MSssl. We also thank Ibolya Anton for the technical assistance. This work was supported by the K 83983 grant from the Hungarian Scientific Research Fund (OTKA) and by the COOP-CT-2005017984 grant from the European Commission's Sixth Framework Programme.

\section{Authors' contributions}

KS-K carried out most of the experimental work and participated in drafting the manuscript. ET constructed some of the plasmids and studied complementation between the plasmid pairs. AK designed the study, participated in the experimental work and wrote the manuscript. All authors read and approved the final manuscript.

Received: 2 March 2012 Accepted: 30 May 2012

Published: 30 May 2012

\section{References}

1. Jeltsch A: Beyond Watson and Crick: DNA methylation and molecular enzymology of DNA methyltransferases. ChemBioChem 2002, 3:274-293.

2. Kumar S, Cheng X, Klimasauskas S, Mi S, Pósfai J, Roberts RJ, Wilson GG: The DNA (cytosine-5) methyltransferases. Nucleic Acids Res 1994, 22:1-10.

3. Pósfai J, Bhagwat AS, Pósfai G, Roberts RJ: Predictive motifs derived from cytosine methyltransferases. Nucleic Acids Res 1989, 17:2421-2435.

4. Wu JC, Santi DV: Kinetic and catalytic mechanism of Hhal methyltransferase. J Biol Chem 1987, 262:4778-4786.

5. Chen L, MacMillan AM, Chang W, Ezaz-Nikpay K, Lane WS, Verdine GL: Direct identification of the active-site nucleophile in a DNA (cytosine-5)methyltransferase. Biochemistry 1991, 30:11018-11025. 
6. Estabrook RA, Lipson R, Hopkins B, Reich N: The coupling of tight DNA binding and base flipping: identification of a conserved structural motif in base flipping enzymes. J Biol Chem 2004, 279:31419-31428.

7. Shieh FK, Reich NO: AdoMet-dependent methyl-transfer: Glu119 is essential for DNA C5-cytosine methyltransferase M.Hhal. J Mol Biol 2007, 373:1157-1168.

8. Darii MV, Cherepanova NA, Subach OM, Kirsanova OV, Rasko T, Slaska-Kiss K, Kiss A, Deville-Bonne D, Reboud-Ravaux M, Gromova ES: Mutational analysis of the CG recognizing DNA methyltransferase Sssl: Insight into enzyme-DNA interactions. Biochim Biophys Acta 2009, 1794:1654-1662.

9. Sankpal UT, Rao DN: Mutational analysis of conserved residues in Hhal DNA methyltransferase. Nucleic Acids Res 2002, 30:2628-2638.

10. Balganesh TS, Reiners L, Lauster R, Noyer-Weidner M, Wilke K, Trautner TA: Construction and use of chimeric SPR/phi3T DNA methyltransferases in the definition of sequence recognizing enzyme regions. EMBO J 1987, 6:3543-3549.

11. Klimasauskas $\mathrm{S}$, Nelson $\mathrm{J}$, Roberts RJ: The sequence specificity domain of cytosine-C5 methylases. Nucleic Acids Res 1991, 19:6183-6190.

12. Klimasauskas S, Kumar S, Roberts RJ, Cheng X: Hhal methyltransferase flips its target base out of the DNA helix. Cell 1994, 76:357-369.

13. Reinisch KM, Chen L, Verdine GL, Lipscomb WN: The crystal structure of Haell methyltransferase covalently complexed to DNA: An extrahelical cytosine and rearranged base pairing. Cell 1995, 82:143-153.

14. Jurkowska RZ, Jurkowski TP, Jeltsch A: Structure and function of mammalian DNA methyltransferases. ChemBioChem 2011, 12:206-222.

15. Song J, Rechkoblit O, Bestor TH, Patel DJ: Structure of DNMT1-DNA complex reveals a role for autoinhibition in maintenance DNA methylation. Science 2011, 331:1036-1040.

16. Karreman C, de Waard A: Agmenellum quadruplicatum M.Aqul, a novel modification methylase. J Bacterio/ 1990, 172:266-272.

17. Pinarbasi H, Pinarbasi E, Hornby D: Recombinant alpha and beta subunits of M.Aqul constitute an active DNA methyltransferase. J Biochem Mol Biol 2002, 35:348-351.

18. Lee KF, Kam KM, Shaw PC: A bacterial methyltransferase M.EcoHK311 requires two proteins for in vitro methylation. Nucleic Acids Res 1995, 23:103-108

19. Pósfai G, Kim SC, Szilák L, Kovács A, Venetianer P: Complementation by detached parts of GGCC-specific DNA methyltransferases. Nucleic Acids Res 1991, 19:4843-4847.

20. Choe W, Chandrasegaran S, Ostermeier M: Protein fragment complementation in M.Hhal DNA methyltransferase. Biochem Biophys Res Commun 2005, 334:1233-1240.

21. Renbaum P, Abrahamove D, Fainsod A, Wilson GG, Rottem S, Razin A: Cloning, characterization, and expression in Escherichia coli of the gene coding for the CpG DNA methylase from Spiroplasma sp. strain MQ1(M. Sssl). Nucleic Acids Res 1990, 18:1145-1152.

22. Klose RJ, Bird AP: Genomic DNA methylation: the mark and its mediators. Trends Biochem Sci 2006, 31:89-97.

23. Xu GL, Bestor TH: Cytosine methylation targetted to pre-determined sequences. Nat Genet 1997, 17:376-378.

24. Li F, Papworth M, Minczuk M, Rohde C, Zhang Y, Ragozin S, Jeltsch A: Chimeric DNA methyltransferases target DNA methylation to specific DNA sequences and repress expression of target genes. Nucleic Acids Res 2007, 35:100-112.

25. Smith AE, Hurd PJ, Bannister AJ, Kouzarides T, Ford KG: Heritable gene repression through the action of a directed DNA methyltransferase at a chromosomal locus. J Biol Chem 2008, 283:9878-9885.

26. Jurkowska RZ, Jeltsch A: Silencing of gene expression by targeted DNA methylation: concepts and approaches. Methods Mol Biol 2010, 649:149-161.

27. Rivenbark AG, Stolzenburg S, Beltran AS, Yuan X, Rots MG, Strahl BD, Blancafort P: Epigenetic reprogramming of cancer cells via targeted DNA methylation. Epigenetics 2012, 7:350-360.

28. Nomura W, Barbas CF 3rd: In vivo site-specific DNA methylation with a designed sequence-enabled DNA methylase. J Am Chem Soc 2007, 129:8676-8677.

29. Meister GE, Chandrasegaran S, Ostermeier M: An engineered split M.Hhalzinc finger fusion lacks the intended methyltransferase specificity. Biochem Biophys Res Commun 2008, 377:226-230.

30. Kiss A, Weinhold E: Functional reassembly of split enzymes on-site: a novel approach for highly sequence-specific targeted DNA methylation. ChemBioChem 2008, 9:351-353.
31. Meister GE, Chandrasegaran S, Ostermeier M: Heterodimeric DNA methyltransferases as a platform for creating designer zinc finger methyltransferases for targeted DNA methylation in cells. Nucleic Acids Res 2010, 38:1749-1759.

32. Koudan EV, Bujnicki JM, Gromova ES: Homology modeling of the CGspecific DNA methyltransferase Sssl and its complexes with DNA and AdoHcy. J Biomol Struct Dyn 2004, 22:339-345.

33. Larkin MA, Blackshields G, Brown NP, Chenna R, McGettigan PA, McWilliam H, Valentin F, Wallace IM, Wilm A, Lopez R, et al: Clustal W and Clustal X version 2.0. Nucleic Acids Res 2007, 23:2947-2948.

34. Guzman LM, Belin D, Carson MJ, Beckwith J: Tight regulation, modulation, and high-level expression by vectors containing the arabinose PBAD promoter. J Bacteriol 1995, 177:4121-4130

35. Tímár E, Venetianer P, Kiss A: In vivo DNA protection by relaxed-specificity Sinl DNA methyltransferase variants. J Bacteriol 2008, 190:8003-8008.

36. van der Gun BTF, Maluszynska-Hoffman M, Kiss A, Arendzen AJ, Ruiters MHJ, McLaughlin PMJ, Weinhold E, Rots MG: Targeted DNA methylation by a DNA methyltransferase coupled to a triple helix forming oligonucleotide to down-regulate the epithelial cell adhesion molecule. Bioconjugate Chem 2010, 21:1239-1245.

37. Chen Y, Li S, Chen T, Hua H, Lin Z: Random dissection to select for protein split sites and its application in protein fragment complementation. Protein Sci 2009, 18:399-409.

38. Paschon DE, Patel ZS, Ostermeier M: Enhanced catalytic efficiency of aminoglycoside phosphotransferase (3')-Ila achieved through protein fragmentation and reassembly. J Mol Biol 2005, 353:26-37.

39. Tan S, Guschin D, Davalos A, Lee YL, Snowden AW, Jouvenot Y, Zhang HS, Howes K, McNamara AR, Lai A, et al: Zinc-finger protein-targeted gene regulation: genomewide single-gene specificity. Proc Natl Acad Sci U S A 2003, 100:11997-12002.

40. Gommans WM, McLaughlin PM, Lindhout BI, Segal DJ, Wiegman DJ, Haisma $\mathrm{HJ}$, van der Zaal BJ, Rots MG: Engineering zinc finger protein transcription factors to downregulate the epithelial glycoprotein-2 promoter as a novel anti-cancer treatment. Mol Carcinog 2007, 46:391-401.

41. Durai S, Mani M, Kandavelou K, Wu J, Porteus MH, Chandrasegaran S: Zinc finger nucleases: custom-designed molecular scissors for genome engineering of plant and mammalian cells. Nucl Acids Res 2005, 33:5978-5990.

42. Durfee T, Nelson R, Baldwin S, Plunkett G, Burland V, Mau B, Petrosino JF, Qin X, Muzny DM, Ayele M, et al: The complete genome sequence of Escherichia coli DH10B: insights into the biology of a laboratory workhorse. J Bacteriol 2008, 190:2597-2606.

43. Sambrook J, Russell DW: The Condensed Protocols. From Molecular Cloning: A Laboratory Manual. Cold Spring Harbor Laboratory Press, Cold Spring Harbor, New York 2006

44. Kunkel TA, Roberts JD, Zakour RA: Rapid and efficient site-specific mutagenesis without phenotypic selection. Methods Enzymol 1987, 154:367-382.

45. Greene PH, Poonian MS, Nussbaum AL, Tobias L, Garfin DE, Boyer HW, Goodman HM: Restriction and modification of a self-complementary octanucleotide containing the EcoRl substrate. J Mol Biol 1975, 99:237-261.

\section{doi:10.1186/1471-2199-13-17}

Cite this article as: Ślaska-Kiss et al.: Complementation between inactive fragments of Sssl DNA methyltransferase. BMC Molecular Biology 2012 13:17.

\section{Submit your next manuscript to BioMed Central and take full advantage of:}

- Convenient online submission

- Thorough peer review

- No space constraints or color figure charges

- Immediate publication on acceptance

- Inclusion in PubMed, CAS, Scopus and Google Scholar

- Research which is freely available for redistribution 